\title{
Variation in the resistin gene is associated with obesity and insulin-related phenotypes in Finnish subjects
}

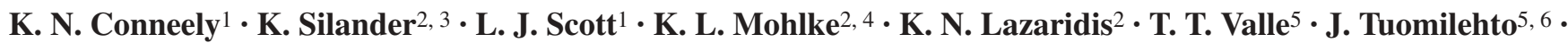 \\ R. N. Bergman ${ }^{7}$ R. M. Watanabe ${ }^{8}$ - T. A. Buchanan ${ }^{9}$ F. S. Collins ${ }^{2} \cdot$ M. Boehnke ${ }^{1}$ \\ ${ }^{1}$ Department of Biostatistics, School of Public Health, University of Michigan, Ann Arbor, USA \\ ${ }^{2}$ Genome Technology Branch, National Human Genome Research Institute, Bethesda, Maryland, USA \\ ${ }^{3}$ Department of Molecular Medicine, National Public Health Institute, Helsinki, Finland \\ ${ }^{4}$ Department of Genetics, University of North Carolina, Chapel Hill, North Carolina, USA \\ ${ }^{5}$ Diabetes and Genetic Epidemiology Unit, Department of Epidemiology and Health Promotion, National Public Health Institute, \\ Helsinki, Finland \\ ${ }^{6}$ Department of Public Health, University of Helsinki, Helsinki, Finland \\ ${ }^{7}$ Department of Physiology and Biophysics, Keck School of Medicine, University of Southern California, Los Angeles, \\ California, USA \\ ${ }^{8}$ Department of Preventive Medicine, Keck School of Medicine, University of Southern California, Los Angeles, California, USA \\ ${ }_{9}^{9}$ Department of Medicine, Division of Endocrinology, Keck School of Medicine, University of Southern California, Los Angeles, \\ California, USA
}

\section{Abstract}

Aims/hypothesis. Resistin is a peptide hormone produced by adipocytes that is present at high levels in sera of obese mice and may be involved in glucose homeostasis through regulation of insulin sensitivity. Several studies in humans have found associations between polymorphisms in the resistin gene and obesity, insulin sensitivity and blood pressure. An association between variation in the resistin gene and Type 2 diabetes has been reported in some, but not all studies. The aim of this study was to analyse variants of the resistin gene for association with Type 2 diabetes and related traits in a Finnish sample.

Methods. In 781 cases with Type 2 diabetes, 187 spouse controls and 222 elderly controls of Finnish origin, we genotyped four previously identified non-coding single-nucleotide polymorphisms (SNPs): $-420 C>G$ from the promoter region, $+156 C>T$ and $+298 G>A$ from intron 2 , and $+1084 G>A$ from the $3^{\prime}$ untranslated region. We then tested whether these
SNPs were associated with Type 2 diabetes and related traits.

Results. The SNPs were not significantly associated with Type 2 diabetes. However, SNPs $-420 C>G$, $+156 C>T$ and $+298 G>A$ and the common haplotype for these three markers were associated with increased values of weight-related traits and diastolic blood pressure in cases, lower weight in elderly control subjects, and lower insulin sensitivity and greater acute insulin response in spouses. Furthermore, the $+1084 G$ allele was associated with lower HDL cholesterol in both cases and controls, higher systolic blood pressure and waist circumference in cases, and greater acute insulin response in spouse controls.

Conclusions/interpretation. Our results add to growing evidence that resistin is associated with variation in weight, fat distribution and insulin resistance.

Keywords Genetics of Type 2 diabetes - Genetic susceptibility $\cdot$ Human genetics $\cdot$ Non-insulin-dependent diabetes mellitus
Received: 22 March 2004 / Accepted: 22 July 2004

Published online: 27 October 2004

(C) Springer-Verlag 2004

M. Boehnke (๘)

Department of Biostatistics, School of Public Health,

University of Michigan, 1420 Washington Heights,

Ann Arbor, MI 48109-2029, USA

E-mail: boehnke@umich.edu

Tel.: +1-734-9361001

Fax: +1-734-7632215
Electronic Supplementary Material

Supplementary material is available in the online version of this article at http://dx.doi.org/10.1007/s00125-004-1537-x

Abbreviations: AIR, acute insulin response to glucose $\cdot \mathrm{DI}$, disposition index $\cdot$ FUSION, Finland-United States Investigation of NIDDM Genetics · LD, linkage disequilibrium $\cdot S_{I}$, insulin sensitivity $\cdot S_{\mathrm{G}}$, glucose effectiveness $\cdot \mathrm{SNP}$, single-nucleotide polymorphism $\cdot$ UTR, untranslated region 


\section{Introduction}

Type 2 diabetes is caused by poor pancreatic beta cell function on a background of insulin resistance. Obesity, especially abdominal and visceral obesity, is commonly associated with, and may cause, insulin resistance in Type 2 diabetes. Signalling molecules secreted by adipose tissue may provide the link between insulin resistance and obesity, and resistin is one such molecule [1]. In murine models, resistin is induced during adipocyte differentiation and its expression decreases upon treatment with insulin sensitizers. Serum levels of resistin are elevated in obese mice, both when the obesity is genetic and when it is the result of a high-fat diet, and administration of resistin impairs glucose tolerance in normal mice. This suggests that resistin links obesity to insulin resistance and Type 2 diabetes [1]. However, other studies of rat and mouse models have associated insulin resistance and obesity with decreased resistin expression [2, 3]. It has recently been reported that mice lacking the resistin gene resemble normal mice in most respects, including body weight, but have better glucose tolerance that stems from reduced hepatic glucose production [4]. Among mice on high-fat diets, the relationship between body weight and blood glucose is weaker in mice lacking resistin than in normal mice, suggesting that the effects of resistin may be mediated by diet and obesity [4].

Human studies have found no relationship between resistin expression and insulin resistance or adiposity $[5,6,7]$, although one study found that resistin mRNA expression decreased during adipogenic differentiation [5]. One possible explanation for these seemingly contradictory findings is that resistin expression may be inhibited by insulin and other factors related to obesity $[8,9]$. Alternatively, resistin may block adipose tissue formation [10], leading to insulin resistance due to a lack of fresh, insulin-sensitive adipocytes [11].

Although the role of resistin in the pathogenesis of Type 2 diabetes has not been fully established, its potential importance is supported by studies of variation in the human resistin gene. Non-coding variants of the gene have been identified $[12,13,14,15,16,17,18$, $19,20,21,22]$ and have been shown to be associated with variables related to weight $[12,13]$, insulin $[14$, 15] and blood pressure [16]. A recent study reported that a polymorphism in the promoter region was associated with resistin mRNA levels in abdominal subcutaneous fat [12]. Associations between resistin polymorphisms and Type 2 diabetes have been reported in some studies [16, 17], but not others [13, 14, 18, 19, 20]. In the present study we genotyped four variants of the resistin gene that have shown evidence of association with Type 2 diabetes-related traits $[12,13,14$, $15,16]$ and tested whether these single-nucleotide polymorphisms (SNPs) were associated with Type 2 diabetes and related quantitative traits.

\section{Subjects and methods}

Subjects. The study group was a Finnish cohort of 781 Type 2 diabetes cases and 409 control subjects from the Finland-United States Investigation of NIDDM Genetics (FUSION) Study (http://fusion.sph.umich.edu) [23]. The FUSION study was designed to identify genetic variants that predispose individuals to Type 2 diabetes and influence intermediate quantitative traits in the Finnish population. The study design and details regarding the determination of diabetes status and diabetes-related traits have been described previously [23]. Cases were unrelated individuals with Type 2 diabetes, almost all from families with at least two recruited Type 2 diabetes siblings. Controls were 187 normoglycaemic spouses of Type 2 diabetes cases and 222 unrelated subjects who had normal glucose tolerance according to OGTTs performed at the ages of 65 and 70 years (elderly controls). Cases, normoglycaemic spouses, and elderly controls are further described elsewhere [24]. Excluded from analysis were: (i) cases who had a first-degree relative with Type 1 diabetes; (ii) cases with uncertain diabetes status; and (iii) cases who took medication expected to influence the traits of interest on the day of examination. Cases were receiving treatment for Type 2 diabetes in the form of diet and, for the majority of subjects, some combination of insulin and oral medication. The majority of cases and control subjects could be characterised as hypertensive, and most of these individuals were being treated with anti-hypertensive drugs. About $30 \%$ of cases and controls were also treated with lipid-lowering drugs. Characteristics of the study subjects, including the rates of different types of treatment and prevalence of hypertension, are summarised in Table 1. Informed consent was obtained from each study participant, and all protocols were approved by the Ethics Committee or Institutional Review Board at each of the participating centres.

Genotyping. We genotyped four previously identified non-coding SNPs: $-420 C>G$ (rs1862513) from the promoter region $[12,13,14,15,17],+156 C>T(\mathrm{rs} 3219177)[13,14,15,17,18$, $21]$ and $+298 G>A(\operatorname{rs} 3745367)[14,17,18,19]$ from intron 2 , and $+1084 G>A\left(\right.$ rs3745368) from the $3^{\prime}$ untranslated region (UTR) on exon $4[16,21]$ of the resistin gene on chromosome 19. These four SNPs were chosen because they had shown evidence of association with Type 2 diabetes-related traits in other study samples $[12,13,14,15,16]$. The SNPs had an allele frequency of $>0.1$ in Caucasians, and comprised four of the six common $(>0.1)$ variations identified in previous studies that screened both coding and regulatory regions of the gene for polymorphisms $[13,14,15,17,18,20,21]$. In order to identify any additional SNPs in coding regions, we screened $760 \mathrm{bp}$ of the promoter region and all four exons in 94 individuals of Finnish origin; however, we found no additional variants with an allele frequency of $>0.1$ [22]. The SNPs were genotyped by homogeneous MassEXTEND reaction using the MassARRAY System (Sequenom, San Diego, Calif., USA) as previously described [25]. Assay details are available from the authors upon request. There were 5644 successful genotypes out of the 5756 attempted (98.1\%). Among 324 blinded duplicate genotype pairs, one discrepancy was observed. The genotype frequencies of all four SNPs were in Hardy-Weinberg equilibrium.

Haplotype assignment. Haplotype frequencies were estimated by the EM algorithm [26] and were used to estimate linkage disequilibrium (LD) between markers. Individual haplotypes were assigned as the pair of haplotypes with the maximum posterior probability computed from the estimated haplotype frequencies. Haplotypes were assigned if the maximum poste- 
Table 1. Characteristics of the subjects by clinical subgroup

\begin{tabular}{|c|c|c|c|}
\hline & $\begin{array}{l}\text { Cases with T2D } \\
(n=781)\end{array}$ & $\begin{array}{l}\text { Spouse controls } \\
(n=187)\end{array}$ & $\begin{array}{l}\text { Elderly controls } \\
(n=222)\end{array}$ \\
\hline $\operatorname{Sex}(M: F)$ & 435:346 & $61: 126$ & 107:115 \\
\hline Age at enrolment (years) & $63.9 \pm 7.5$ & $61.7 \pm 7.8$ & $70.0 \pm 0.3$ \\
\hline Age at diagnosis (years) & $50.9 \pm 8.3$ & - & - \\
\hline Diabetes duration (years) & $13.1 \pm 7.4$ & - & - \\
\hline BMI $\left(\mathrm{kg} / \mathrm{m}^{2}\right)$ & $29.9 \pm 4.6$ & $28.5 \pm 4.5$ & $27.1 \pm 4.0$ \\
\hline Fasting plasma glucose $(\mathrm{mmol} / \mathrm{l})$ & $10.3 \pm 3.3$ & $5.3 \pm 0.7$ & $5.0 \pm 0.5$ \\
\hline Fasting serum insulin $(\mathrm{pmol} / \mathrm{l})$ & $114.7 \pm 95.7$ & $76.9 \pm 49.8$ & $65.3 \pm 32.5$ \\
\hline Treated for T2D with insulin (\%) & 48.0 & - & - \\
\hline Treated for T2D with tablets $(\%)^{\mathrm{a}}$ & 67.2 & - & - \\
\hline Treated for T2D with diet only (\%) & 7.8 & - & - \\
\hline Hypertension $(\%)^{\mathrm{b}}$ & 80.1 & 66.7 & 64.6 \\
\hline Treated with anti-hypertensive drugs (\%) & 71.6 & 50.4 & 48.3 \\
\hline Treated with counteracting lipids (\%) & 30.1 & 33.9 & 29.4 \\
\hline
\end{tabular}

Values are means \pm SD or percentages. a Treatment with sulphonylureas or metformin; ${ }^{b}$ individuals were characterised as hypertensive if they met at least one of the following criteria:

rior probability exceeded 0.9 , which was true in all cases. A probability cut-off point of 0.99 resulted in the exclusion of 43 individuals (3.6\% of our sample) but did not yield any meaningful differences in the results. In the results reported here, we used 0.9 as our cut-off point, which allowed us to use the entire sample.

Association analyses. Associations between Type 2 diabetes and SNPs and haplotypes were assessed using chi square tests of independence and logistic regression adjusting for age and sex. Association tests were performed for: (i) the entire sample; (ii) the control group restricted to either spouses or elderly controls; and (iii) individuals with $\mathrm{BMI}<30$, BMI $\geq 30$ and $\mathrm{BMI} \geq 35$.

Associations between quantitative traits and SNPs and haplotypes were tested using ANOVA with adjustment for age and sex. Additional tests with adjustment for BMI were performed for the non-weight-related traits. We assumed that the alleles acted additively, hence all of our ANOVA models included the number of copies of the allele or haplotype as a covariate. Traits were transformed to approximate normality when necessary.

We analysed fourteen traits for all subjects: BMI, WHR, waist circumference, weight at enrolment, self-reported maximum lifetime weight, fasting plasma glucose, fasting serum insulin, total cholesterol, HDL cholesterol, HDL cholesterol : total cholesterol ratio, LDL cholesterol, total triglycerides and systolic and diastolic blood pressure. We measured and analysed three additional traits for Type 2 diabetes subjects and spouse controls: self-reported weight at age 20 years, weight change since age 20 years (enrolment weight-weight at 20 years), and maximum lifetime weight change since age 20 years (maximum lifetime weight-weight at 20). We measured and analysed fasting C-peptide concentrations for cases alone, and 2-h glucose and insulin levels for controls alone. We analysed insulin sensitivity $\left(S_{I}\right)$, glucose effectiveness $\left(S_{G}\right)$, acute insulin response to glucose (AIR), and the disposition index $\left(\mathrm{DI}=\mathrm{S}_{\mathrm{I}} \times \mathrm{AIR}\right)$ for spouse controls who underwent a tolbutamide-modified frequently sampled intravenous glucose tolerance test.

Because of the large number of comparisons (a total of 18 for cases, 23 for spouses and 16 for elderly controls) we performed permutation tests to estimate the probability of observ- (i) systolic blood pressure $\geq 160 \mathrm{~mm} \mathrm{Hg}$; (ii) diastolic blood pressure $\geq 95 \mathrm{~mm} \mathrm{Hg}$; or (iii) receiving treatment with antihypertensive drugs. T2D: Type 2 diabetes ing a similar number of associated traits due to chance alone. For each study group (cases, spouses, and elderly control subjects), we created 10,000 permuted samples by randomly shuffling genotypes across the sample (leaving the vector of correlated traits, including sex and age, intact). For each permuted sample, we performed ANOVA tests of association for all traits. We then compared the pattern of trait associations in the permuted samples to the original cluster of traits significant at the 0.05 level. We computed the overall $p$ value for each study group as the proportion of permuted samples having at least as many traits with $p$ values less than or equal to the least significant $p$ value in the original cluster of significant traits in that group.

\section{Results}

Disease association. Allele frequencies for the four SNPs did not differ significantly between Type 2 diabetes cases and the combined controls (Table 2), nor were there significant differences when subjects were stratified by BMI or when the control group was restricted to spouses or elderly controls $(p>0.15)$.

Haplotype analysis. Three of the four SNPs $(-420 C>$ $G,+156 C>T$, and $+298 G>A$ ) had similar minor allele frequencies of $\sim 0.25$ and were in strong LD, with $R^{2} \approx 0.8$. These SNPs formed two predominant haplotypes: $C C G(72 \%)$ and GTA $(22 \%)$. The minor allele of SNP $+1084 G>A$ was rarer, with a frequency of 0.02 , and did not appear in either of the major haplotypes. Frequencies of the common haplotypes were not significantly different between cases and controls (data not shown).

Trait associations. Table 3 shows the mean values for significantly associated traits $(p<0.05$, adjusted for sex and age) for subjects with 0,1 or 2 copies of the common $C C G$ haplotype (means for all traits can be found 
Table 2. Allele counts and frequencies among Type 2 diabetes cases and control subjects

\begin{tabular}{|c|c|c|c|c|c|}
\hline \multirow[t]{2}{*}{ SNP } & \multirow[t]{2}{*}{ Allele } & \multicolumn{2}{|c|}{ Allele counts/total (frequency) } & \multirow[t]{2}{*}{$p$ value ${ }^{\mathrm{a}}$} & \multirow{2}{*}{$\begin{array}{l}\text { Adjusted } \\
p \text { value }^{b}\end{array}$} \\
\hline & & Cases & Controls & & \\
\hline$+156 C>T$ & $T$ & $368 / 1590(0.23)$ & $190 / 824(0.23)$ & 0.96 & 0.99 \\
\hline$+298 G>A$ & $A$ & $402 / 1586(0.25)$ & $205 / 826(0.24)$ & 0.78 & 0.77 \\
\hline$+1084 G>A$ & $A$ & $31 / 1550(0.02)$ & $17 / 806(0.02)$ & 0.86 & 0.56 \\
\hline
\end{tabular}

a Based on chi square test of independence; $b$ based on logistic regression, adjusted for age and sex

Table 3. Traits associated with the $C C G$ haplotype

\begin{tabular}{|c|c|c|c|c|}
\hline \multirow[t]{2}{*}{ Trait } & \multicolumn{3}{|c|}{ Number of copies of the $C C G$ haplotype } & \multirow[t]{2}{*}{$p$ value $^{\mathrm{a}}$} \\
\hline & 0 & 1 & 2 & \\
\hline Waist circumference $(\mathrm{cm})$ & $99.5 \pm 8.5$ & $101.3 \pm 10.8$ & $103.4 \pm 12.6$ & 0.001 \\
\hline Weight $(\mathrm{kg})$ & $80.8 \pm 10.1$ & $83.2 \pm 14.1$ & $84.8 \pm 15.0$ & 0.005 \\
\hline Weight change $(\mathrm{kg})^{\mathrm{b}}$ & $16.4 \pm 10.8$ & $19.5 \pm 11.9$ & $21.1 \pm 14.2$ & 0.005 \\
\hline BMI $\left(\mathrm{kg} / \mathrm{m}^{2}\right)$ & $29.1 \pm 3.6$ & $29.5 \pm 4.5$ & $30.4 \pm 4.7$ & $0.019 \mathrm{c}$ \\
\hline Elderly control subjects & $n=17$ & $n=97$ & $n=107$ & \\
\hline Weight (kg) & $80.4 \pm 17.1$ & $75.1 \pm 13.5$ & $73.5 \pm 11.9$ & $0.032^{\mathrm{d}}$ \\
\hline Maximum weight (kg) & $84.5 \pm 18.8$ & $78.6 \pm 13.7$ & $77.6 \pm 12.8$ & 0.051 \\
\hline Spouse control subjects & $n=12$ & $n=74$ & $n=101$ & \\
\hline $\mathrm{S}_{\mathrm{I}}\left(\times 10^{5} \mathrm{~min}^{-1} \cdot[\mathrm{pmol} / 1]^{-1}\right)$ & $8.27 \pm 6.78$ & $6.47 \pm 3.48$ & $5.23 \pm 2.60$ & 0.013 \\
\hline
\end{tabular}

Values are means \pm SD. For data on all traits tested, please see ESM Table 1. a Based on a test of effects of the number of copies of the $C C G$ haplotype on the normal-transformed value of the trait, adjusted for age and sex; ${ }^{b}$ current weight minus self-reported weight at age 20 years; ${ }^{c}$ probability of observing at least six traits with $p \leq 0.019$ in Type 2 diabetes cases is
0.002 (estimated from permutation test); d probability of observing at least one trait with $p \leq 0.032$ in elderly control subjects is 0.338 (estimated from permutation test); e probability of observing at least two traits with $p \leq 0.016$ in spouse controls is 0.097 (estimated from permutation test)

unlikely to be due to chance alone $(p=0.002)$. For elderly controls $(p=0.338)$ and spouses $(p=0.097)$, the observed associations could be explained by chance.

Table 4 presents mean trait values by genotype for all traits that were significantly associated $(p<0.05)$ with the 3' UTR SNP $(+1084 G>A)$ (means for all traits are reported in Table 2 of the ESM). Type 2 diabetes subjects with $G / G$ rather than $A / G$ genotypes had higher systolic blood pressures $(p=0.024)$ and mean waist circumferences $(p=0.026)$ and lower levels of HDL cholesterol $(p=0.040)$. Elderly controls with the $G / G$ genotype had lower HDL cholesterol $(p=0.006)$ and HDL : total cholesterol ratios $(p=0.032$ ). Among spouse controls, $G / G$ subjects had significantly lower HDL cholesterol $(p=0.005)$ and greater AIR $(p=0.003)$, weight change $(p=0.041)$ and DI ( $p=0.041)$ than $A / G$ subjects. Again, adjusting for BMI in addition to sex and age did not change the observed pattern of trait associations. Permutation tests showed that for each of the three groups tested, the probability of observing at least as many associated traits due to chance exceeded 0.05 but was still sug- 
Table 4. Traits associated with SNP $+1084 G>A$

\begin{tabular}{|c|c|c|c|}
\hline \multirow[t]{2}{*}{ Trait } & \multicolumn{2}{|c|}{ Genotype at $+1084 G>A$} & \multirow[t]{2}{*}{$p$ value } \\
\hline & $A / G$ & $G / G$ & \\
\hline Type 2 diabetes cases & $n=31$ & $n=742$ & \\
\hline Systolic BP (mm Hg) & $143 \pm 14$ & $153 \pm 22$ & 0.024 \\
\hline Waist circumference $(\mathrm{cm})$ & $99.2 \pm 8.3$ & $102.4 \pm 11.8$ & 0.026 \\
\hline HDL cholesterol $(\mathrm{mmol} / \mathrm{l})$ & $1.20 \pm 0.30$ & $1.10 \pm 0.31$ & $0.040^{\mathrm{b}}$ \\
\hline HDL : total cholesterol ratio & $0.219 \pm 0.053$ & $0.200 \pm 0.064$ & 0.051 \\
\hline Elderly control subjects & $n=8$ & $n=208$ & \\
\hline HDL cholesterol $(\mathrm{mmol} / \mathrm{l})$ & $1.78 \pm 0.46$ & $1.40 \pm 0.30$ & 0.006 \\
\hline HDL : total cholesterol ratio & $0.281 \pm 0.063$ & $0.237 \pm 0.052$ & $0.032^{c}$ \\
\hline Spouse control subjects & $n=9$ & $n=178$ & \\
\hline AIR (pmol/l×8 min) & $944 \pm 805$ & $2403 \pm 1653$ & 0.003 \\
\hline $\mathrm{HDL}(\mathrm{mmol} / \mathrm{l})$ & $1.67 \pm 0.31$ & $1.31 \pm 0.36$ & $0.005^{\mathrm{d}}$ \\
\hline Weight change $(\mathrm{kg})$ & $10.0 \pm 7.4$ & $16.8 \pm 11.0$ & 0.041 \\
\hline DI & $6184 \pm 4949$ & $12503 \pm 8985$ & $0.041^{\mathrm{f}}$ \\
\hline
\end{tabular}

Values are means \pm SD. For data on all traits tested, please see ESM Table 2. a based on a test of effects of the presence of the minor allele on the normal-transformed value of the trait, adjusted for age and sex; ${ }^{b}$ probability of observing at least three traits with $p \leq 0.040$ in Type 2 diabetes cases is 0.076 (estimated from permutation test); ${ }^{c}$ probability of observing at least two traits with $p \leq 0.032$ in elderly control subjects is 0.125 (estimated from permutation test); ${ }^{d}$ probability of observing at least two traits with $p \leq 0.005$ in spouse controls is 0.025 (estimated from permutation test); e current weight minus self-reported weight at age 20 years; ${ }^{\mathrm{f}}$ probability of observing at least four traits with $p \leq 0.041$ in spouse controls is 0.063 (estimated from permutation test) gestive ( 0.076 for cases, 0.125 for elderly controls and 0.063 for spouses). The two most significant traits for spouses (AIR and HDL) had $p$ values less than or equal to 0.005 , which would only be expected by chance $2.5 \%$ of the time.

Cases received treatment for Type 2 diabetes in the form of diet, medication and insulin, and many subjects were hypertensive or were taking other medications that could affect the traits studied (see Subjects and methods section and Table 1). In order to verify that these inter-subject differences were not responsible for the observed trait associations, we tested whether the resistin variants were associated with hypertension, treatment for hypertension and dyslipidaemia or type of treatment for diabetes. No significant associations were found, indicating that the trait associations described above were not simply artefacts of associations of resistin with other diseases or with type of treatment.

\section{Discussion}

Our results provide evidence that variants in the resistin gene are related to obesity and insulin resistance. The $C C G$ haplotype formed by SNPs $-420 C>G,+156 C>T$ and $+298 G>A$ was associated with increased adiposity and weight gain during adult life in cases and with increased insulin resistance in spouse controls; however, $C C G$ was associated with lower body weight in elderly controls. The seemingly contradictory results for these SNPs are not unique to the FUSION study. Of the five studies that have anal- ysed SNP $-420 C>G[12,13,14,15,17]$, three reported significant associations with quantitative traits, all in non-Type 2 diabetes subjects. In two studies, the $C$ allele was associated with reductions in weight-related variables $[12,13]$. In a third study, the $C / C$ genotype of SNP $-420 C>G$ was associated with reduced $\mathrm{S}_{\mathrm{I}}$, and the $C / C$ genotypes of both SNP $-420 C>G$ and SNP $+156 C>T$ were associated with reduced $\mathrm{S}_{\mathrm{I}}$ in interaction with BMI [14]. In a functional study, the $G / G$ genotype of SNP $-420 C>G$ was associated with greater promoter activity in adipocytes and, among 58 overweight and obese subjects, resistin mRNA was present in abdominal subcutaneous fat at significantly higher levels in $G / G$ homozygotes [12]. Together, these findings suggest a complex relationship between the resistin genotype and phenotypes related to insulin reistance and obesity.

Whether the complex and sometimes contradictory findings in this study and others are the result of interactions with other genes or environmental factors that differ between cases and controls remains to be determined. Environmental factors such as treatment could pose serious problems in studies of this kind. As documented in the Results section, the resistin variants studied here were not associated with the type of treatment for Type 2 diabetes, hypertension, or medication for hypertension or dyslipidaemia. Since none of these factors were associated with the SNPs in this study, they are probably not responsible for the associations observed. However, environmental factors that affect the phenotypes studied are likely to reduce the power to detect association in general and could explain why certain traits are associated with variation in the re- 
sistin gene in some studies but not others. Meta-analyses or studies with larger sample sizes may be necessary to resolve the complicated and often contradictory pattern of traits associated with resistin variants.

Another reason for trait associations that differ between specific groups of cases and controls could be that the important variant is in LD with different alleles of the same SNP for different groups. Since all of the SNPs studied here are in regulatory regions, it is possible that they act as proxies for an undetected coding SNP, or perhaps another regulatory SNP. SNP $-420 C>G$ has been shown to be associated with levels of resistin mRNA in overweight subjects [12], which may suggest that the variant of interest is in the promoter. Additional screening for regulatory variants would help to resolve this issue, as would functional studies of these variants.

We observed several associations with phenotypes other than obesity and insulin resistance. Diastolic blood pressure increased with the number of copies of the $C C G$ haplotype in Type 2 diabetes cases. Systolic blood pressure was elevated in cases with the $G / G$ genotype at SNP $+1084 G>A$. In both instances, the genotype associated with higher blood pressure was also associated with greater obesity in cases, suggesting that the effect of genotype on blood pressure may be mediated by obesity. For SNP $+1084 G>A$, HDL cholesterol was lower in subjects with the $G / G$ genotype in all study groups, but $G / G$ was not consistently associated with increased weight. This suggests that variants in the resistin gene could affect HDL metabolism directly or through factors other than obesity.

The lack of an association between resistin genotypes and Type 2 diabetes may reflect the dependence of the pathogenesis of diabetes on dual defects in insulin resistance and insulin secretion [27]. In nonType 2 diabetes subjects, obesity and insulin resistance are normally balanced by increased insulin secretion $[27,28]$. The associations between $C C G$ and low $S_{I}$ and high AIR in spouse controls may be a manifestation of this phenomenon. Genes that promote insulin resistance in certain individuals may provide a background in which Type 2 diabetes can develop, while other genes may determine whether an individual cannot maintain high levels of insulin secretion and thus develops Type 2 diabetes on that background. Our failure to find associations between Type 2 diabetes and variants in the resistin gene could suggest that resistin plays a role in determining the insulin resistance background but has little impact on insulin secretion or beta cell function. However, our analysis of the DI (assessed only in spouse controls), which is used as an indicator of the ability of beta cells to compensate for insulin resistance, did yield marginal evidence of an association.

In conclusion, our results from a sample of Finnish Type 2 diabetes cases and controls provide evidence that non-coding variants in the resistin gene are asso- ciated with variation in obesity and insulin resistance, which are two traits that predispose individuals to Type 2 diabetes. Associations were also found between the SNPs and blood pressure and HDL cholesterol, traits that have been related to insulin resistance and obesity in many studies. These results suggest that variation in the resistin gene is probably not directly involved in the beta cell dysfunction that causes Type 2 diabetes; however, it may play an important role in the pathogenesis of obesity and insulin resistance on which Type 2 diabetes develops.

Acknowledgements. K. N. Conneely and K. Silander contributed equally to this work. The FUSION study is made possible by intramural funds from the National Human Genome Research Institute (project no. OH95-C-N030) and by National Institutes of Health grants DK62370 and HG00376 to M. Boehnke. We thank G. Abecasis for helpful discussions, and N. Riebow and P. White for technical assistance. K. N. Conneely was supported by National Institutes of Health Training Grant HG00040. K. L. Mohlke is the recipient of a Burroughs Wellcome Career Award in the Biomedical Sciences. K. N. Lazaridis was supported by the Mayo Clinic and Foundation (Rochester, Minn., USA). J. Tuomilehto has been partially supported by the Academy of Finland (grant numbers 38387 and 46558). R. N. Bergman was supported by National Institutes of Health grants DK27619 and DK29867. R. M. Watanabe is supported by a grant from the American Diabetes Association. We thank the many Finnish subjects who volunteered to participate in the FUSION study.

\section{References}

1. Steppan CM, Bailey ST, Bhat S et al. (2001) The hormone resistin links obesity to diabetes. Nature 409:307-312

2. Way JM, Gorgun CZ, Tong Q et al. (2001) Adipose tissue resistin expression is severely suppressed in obesity and stimulated by peroxisome proliferator-activated receptor gamma agonists. J Biol Chem 276:25651-25653

3. Juan CC, Au LC, Fang VS et al. (2001) Suppressed gene expression of adipocyte resistin in an insulin-resistant rat model probably by elevated free fatty acids. Biochem Biophys Res Commun 289:1328-1333

4. Banerjee RR, Rangwala SM, Shapiro JS et al. (2004) Regulation of fasted blood glucose by resistin. Science 303:1195-1198

5. Janke J, Engeli S, Gorzelniak K, Luft FC, Sharma AM (2002) Resistin gene expression in human adipocytes is not related to insulin resistance. Obes Res 10:1-5

6. Nagaev I, Smith U (2001) Insulin resistance and type 2 diabetes are not related to resistin expression in human fat cells or skeletal muscle. Biochem Biophys Res Commun 285:561-564

7. Savage DB, Sewter CP, Klenk ES et al. (2001) Resistin/ Fizz3 expression in relation to obesity and peroxisome proliferator-activated receptor-gamma action in humans. Diabetes 50:2199-2202

8. Fasshauer M, Klein J, Neumann S, Eszlinger M, Paschke R (2001) Tumor necrosis factor alpha is a negative regulator of resistin gene expression and secretion in 3T3-L1 adipocytes. Biochem Biophys Res Commun 288:1027-1031

9. Haugen F, Jorgensen A, Drevon CA, Trayhurn P (2001) Inhibition by insulin of resistin gene expression in 3T3-L1 adipocytes. FEBS Lett 507:105-108 
10. Kim KH, Lee K, Moon YS, Sul HS (2001) A cysteine-rich adipose tissue-specific secretory factor inhibits adipocyte differentiation. J Biol Chem 276:11252-11256

11. Banerjee RR, Lazar MA (2003) Resistin: molecular history and prognosis. J Mol Med 81:218-226

12. Smith SR, Bai F, Charbonneau C, Janderova L, Argyropoulos G (2003) A promoter genotype and oxidative stress potentially link resistin to human insulin resistance. Diabetes 52: 1611-1618

13. Engert JC, Vohl MC, Williams SM et al. (2002) 5' flanking variants of resistin are associated with obesity. Diabetes 51:1629-1634

14. Wang H, Chu WS, Hemphill C, Elbein SC (2002) Human resistin gene: molecular scanning and evaluation of association with insulin sensitivity and type 2 diabetes in Caucasians. J Clin Endocrinol Metab 87:2520-2524

15. Pizzuti A, Argiolas A, Di Paola R et al. (2002) An ATG repeat in the $3^{\prime}$-untranslated region of the human resistin gene is associated with a decreased risk of insulin resistance. J Clin Endocrinol Metab 87:4403-4406

16. Tan MS, Chang SY, Chang DM, Tsai JC, Lee YJ (2003) Association of resistin gene $3^{\prime}$-untranslated region $+62 \mathrm{G}>\mathrm{A}$ polymorphism with type 2 diabetes and hypertension in a Chinese population. J Clin Endocrinol Metab $88: 1258-1263$

17. Ma X, Warram JH, Trischitta V, Doria A (2002) Genetic variants at the resistin locus and risk of type 2 diabetes in Caucasians. J Clin Endocrinol Metab 87:4407-4410

18. Osawa H, Onuma H, Murakami A et al. (2002) Systematic search for single nucleotide polymorphisms in the resistin gene: the absence of evidence for the association of three identified single nucleotide polymorphisms with Japanese type 2 diabetes. Diabetes 51:863-866

19. Ochi M, Osawa H, Onuma H et al. (2003) The absence of evidence for major effects of the frequent SNP $+299 \mathrm{G}>\mathrm{A}$ in the resistin gene on susceptibility to insulin resistance syndrome associated with Japanese type 2 diabetes. Diabetes Res Clin Pract 61:191-198

20. Sentinelli F, Romeo S, Arca M et al. (2002) Human resistin gene, obesity, and type 2 diabetes: mutation analysis and population study. Diabetes 51:860-862

21. Cao H, Hegele RA (2001) Single nucleotide polymorphisms of the resistin (RSTN) gene. J Hum Genet 46: 553-555

22. Urbanek M, Du Y, Silander K et al. (2003) Variation in resistin gene promoter not associated with polycystic ovary syndrome. Diabetes 52:214-217

23. Valle T, Tuomilehto J, Bergman RN et al. (1998) Mapping genes for NIDDM. Design of the Finland-United States Investigation of NIDDM Genetics (FUSION) Study. Diabetes Care 21:949-958

24. Silander K, Mohlke KL, Scott LJ et al. (2004) Genetic variation near the hepatocyte nuclear factor-4 alpha gene predicts susceptibility to type 2 diabetes. Diabetes 53: 1141-1149

25. Mohlke KL, Erdos MR, Scott LJ et al. (2002) Highthroughput screening for evidence of association by using mass spectrometry genotyping on DNA pools. Proc Natl Acad Sci USA 99:16928-16933

26. Dempster AP, Laird NM, Rubin DB (1977) Maximum likelihood estimation from incomplete data via the EM algorithm. J R Stat Soc 39:1-38

27. Weyer C, Bogardus C, Mott DM, Pratley RE (1999) The natural history of insulin secretory dysfunction and insulin resistance in the pathogenesis of type 2 diabetes mellitus. J Clin Invest 104:787-794

28. Bergman, RN (1989) Toward physiological understanding of glucose tolerance. Minimal-model approach. Diabetes $38: 1512-1527$ 